\title{
Myrmenaphthol A, Isolated From a Hawaiian Sponge of Genus Myrmekioderma
}

Stephen M. Parrish ${ }^{\dagger}$, Ram P. Neupane ${ }^{\dagger}$, Mary Kay Harper ${ }^{\ddagger}$, John Head, ${ }^{\dagger}$ Philip G. Williams ${ }^{\dagger, *}$

${ }^{\dagger}$ Department of Chemistry, University of Hawaii at Manoa, Honolulu Hawaii, 96822.

${ }^{\ddagger}$ Department of Medicinal Chemistry, University of Utah, Salt Lake City, Utah, 84112 


\section{TABLE OF CONTENTS}

Page

Figure S2 ${ }^{13} \mathrm{C}$ NMR Spectrum (125 MHz) of Myrmenaphthol A (1) in MeOH- $d_{4}$

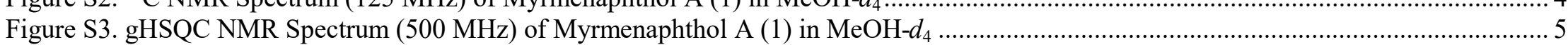

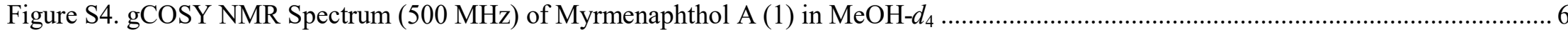

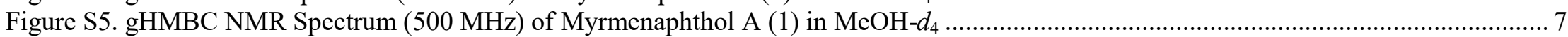

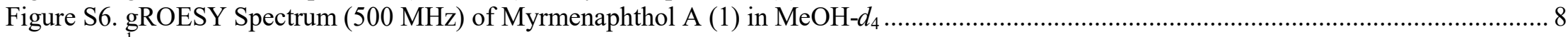

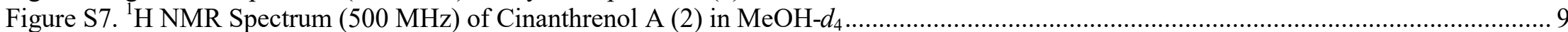

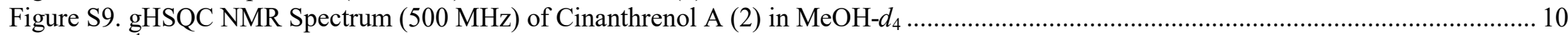

Figure 10: ${ }^{1} \mathrm{H}$ NMR Spectrum of Impure 3,4-dihydroxypregna-5,17-diene-10,2-carbolactone (3) $\left(\mathrm{CD}_{3} \mathrm{OD}, 500 \mathrm{MHz}\right)$................................. 11

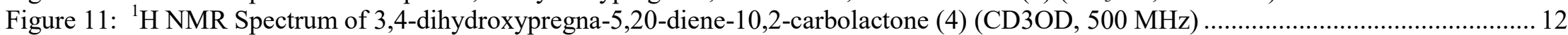

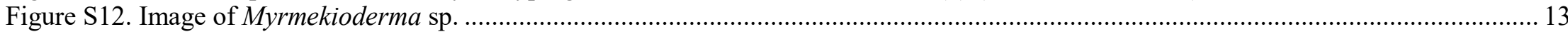

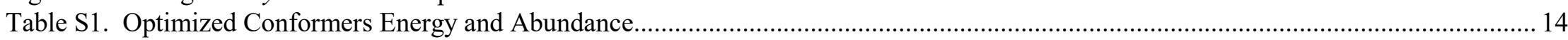

Table S2. Cartesian Coordinates For The Re-Optimized Conformer 1 Of Compound (1) ........................................................................ 15

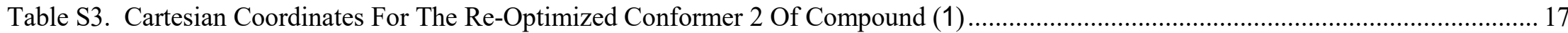

Table S4. Cartesian Coordinates For The Re-Optimized Conformer 3 Of Compound (1) ...........................................................................19

Table S5. Cartesian Coordinates For The Re-Optimized Conformer 4 Of Compound (1) ...........................................................................2 
Figure S1. ${ }^{1} \mathrm{H}$ NMR Spectrum $(500 \mathrm{MHz})$ of Myrmenaphthol A (1) in $\mathrm{MeOH}-d_{4}$

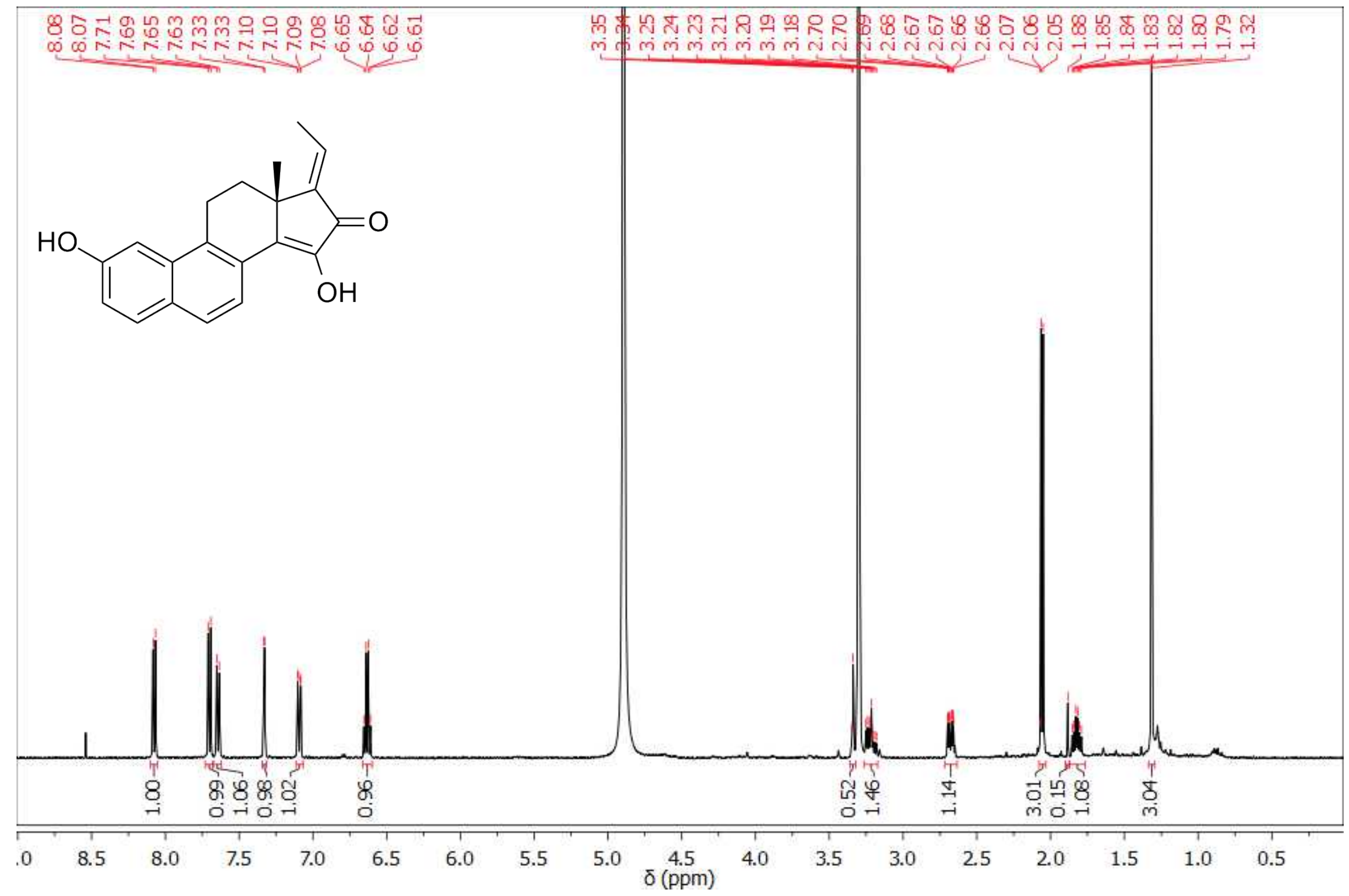


Figure S2. ${ }^{13} \mathrm{C}$ NMR Spectrum (125 MHz) of Myrmenaphthol A (1) in $\mathrm{MeOH}-d_{4}$

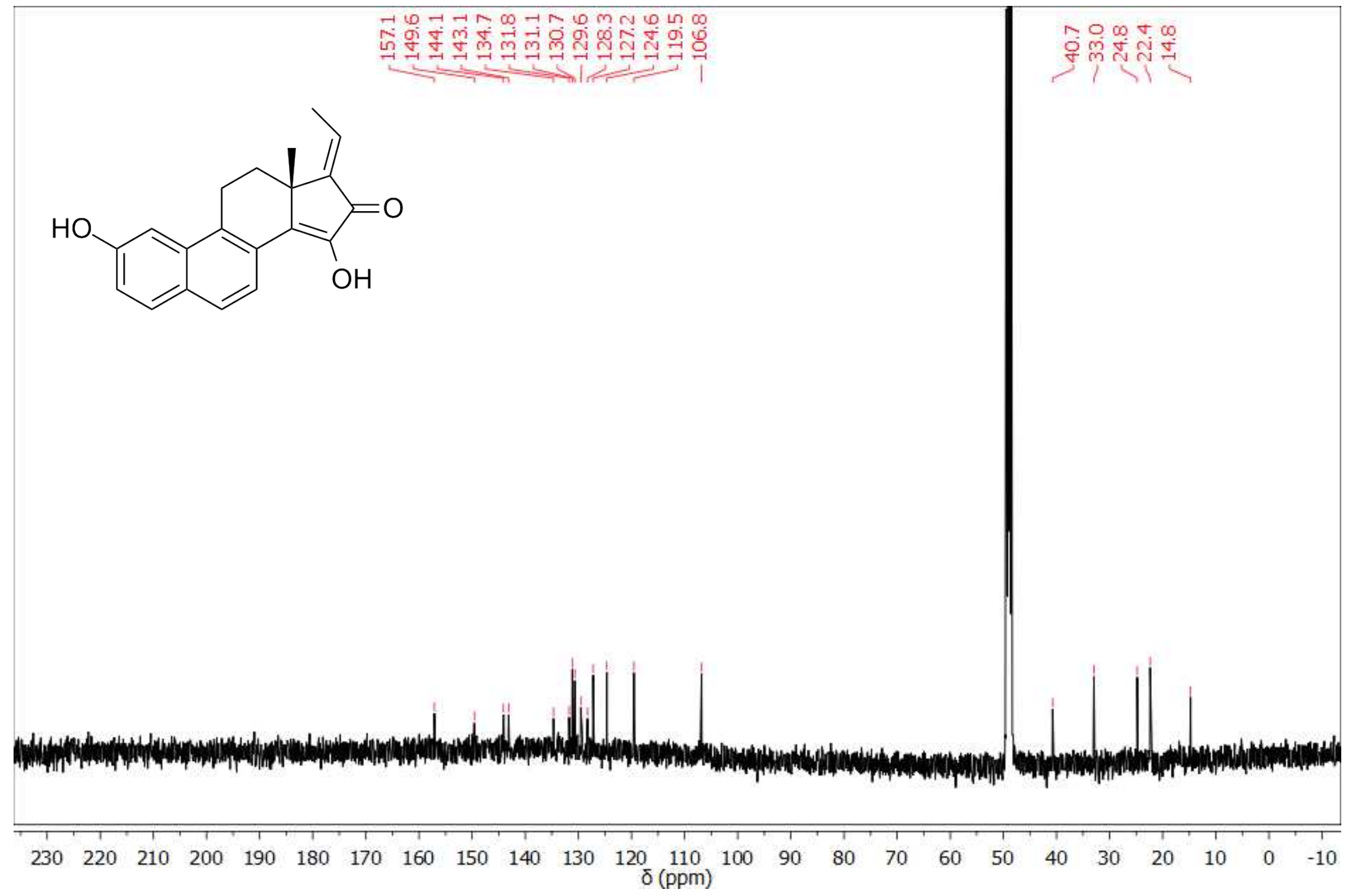


Figure S3. gHSQC NMR Spectrum (500 MHz) of Myrmenaphthol A (1) in MeOH- $d_{4}$

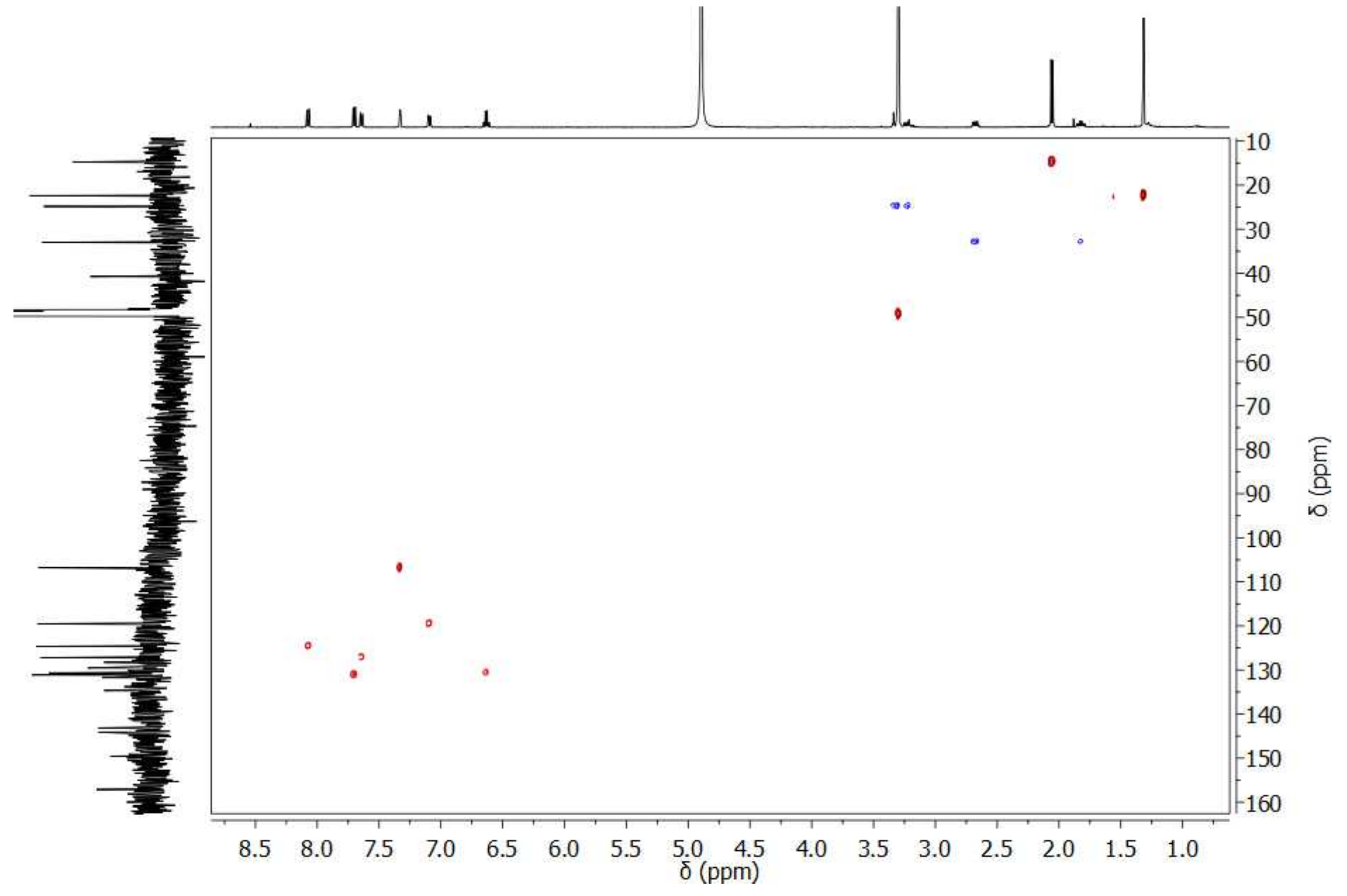


Figure S4. gCOSY NMR Spectrum (500 MHz) of Myrmenaphthol A (1) in MeOH- $d_{4}$

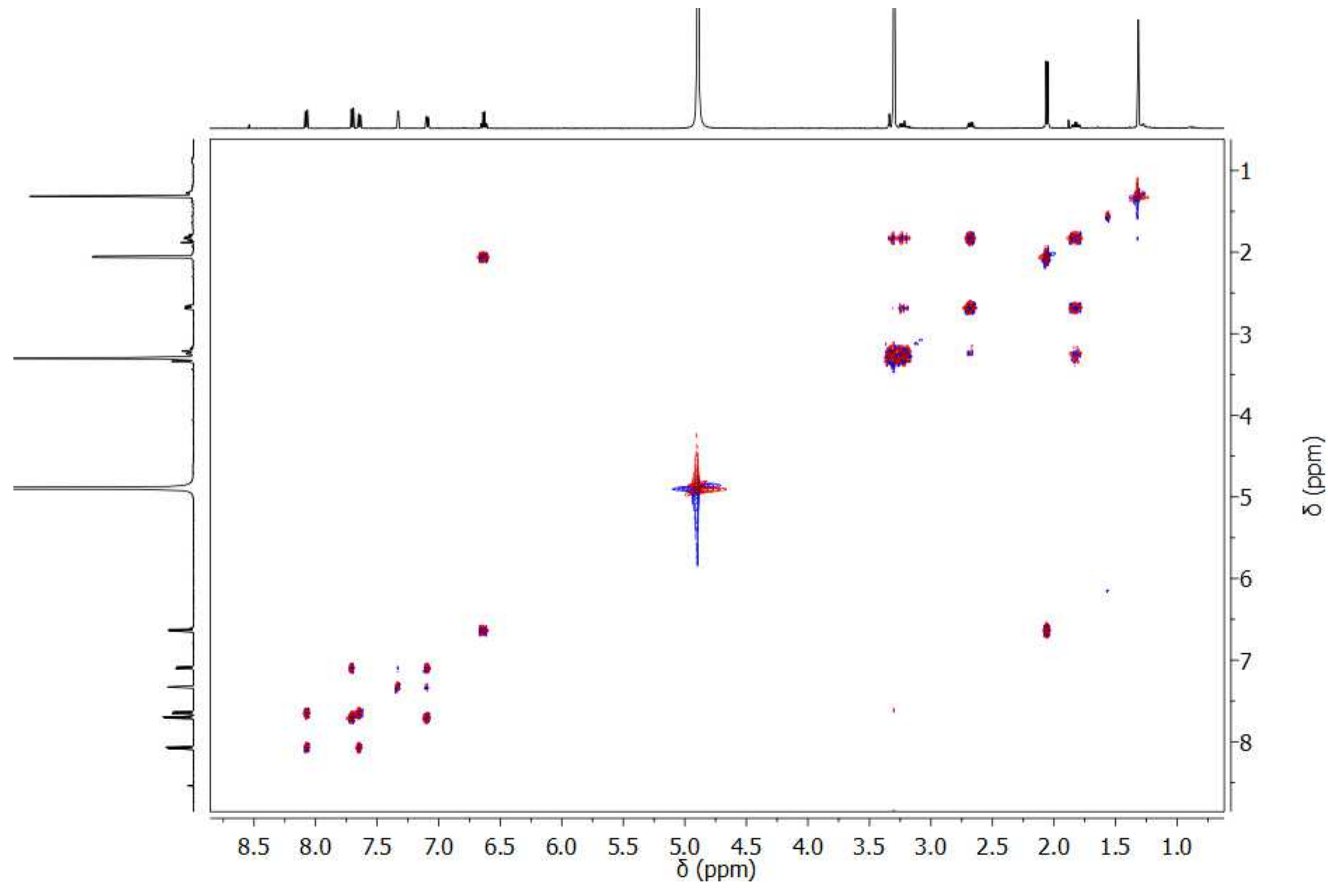


Figure S5. gHMBC NMR Spectrum (500 MHz) of Myrmenaphthol A (1) in MeOH- $d_{4}$

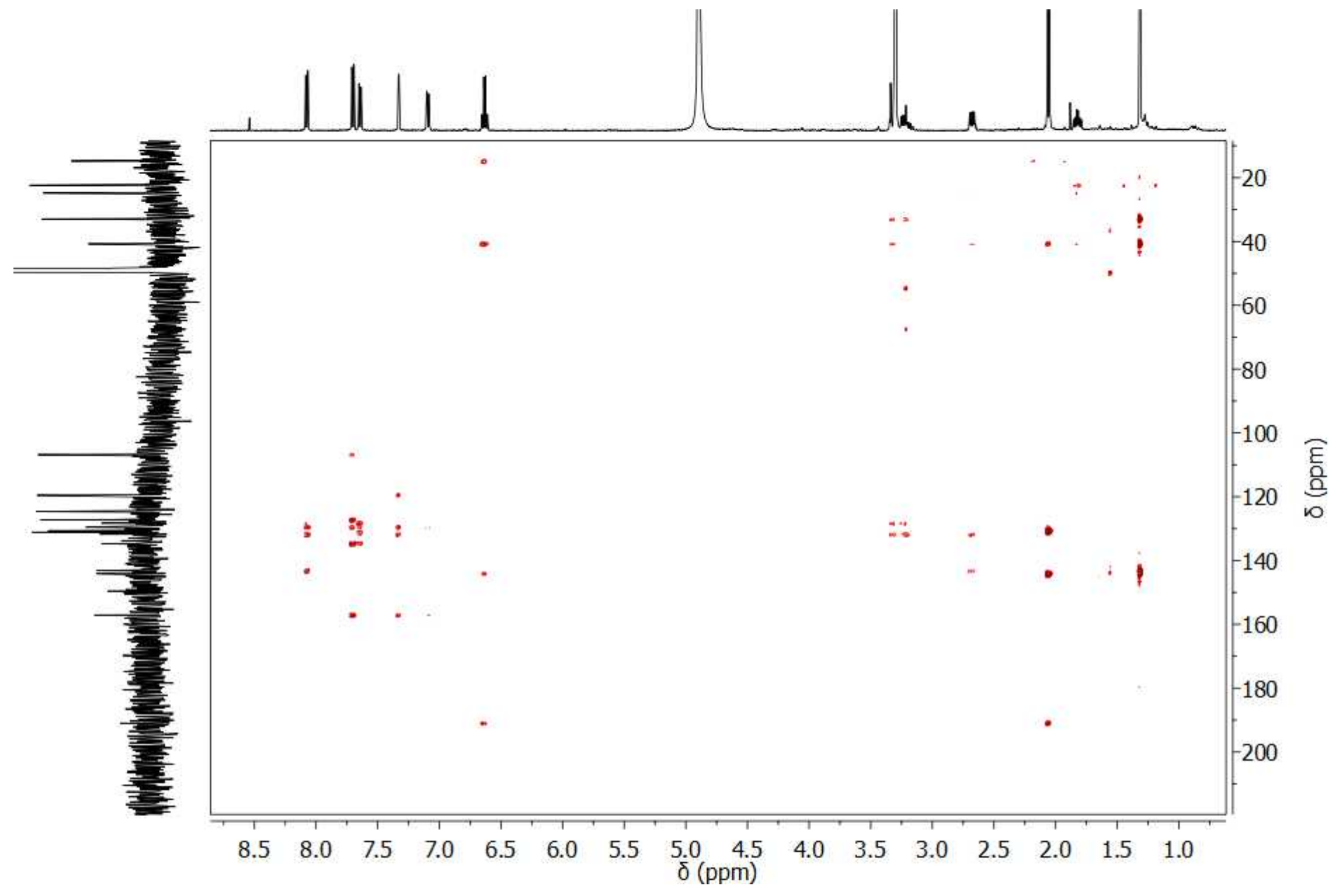


Figure S6. gROESY Spectrum (500 MHz) of Myrmenaphthol A (1) in MeOH- $d_{4}$

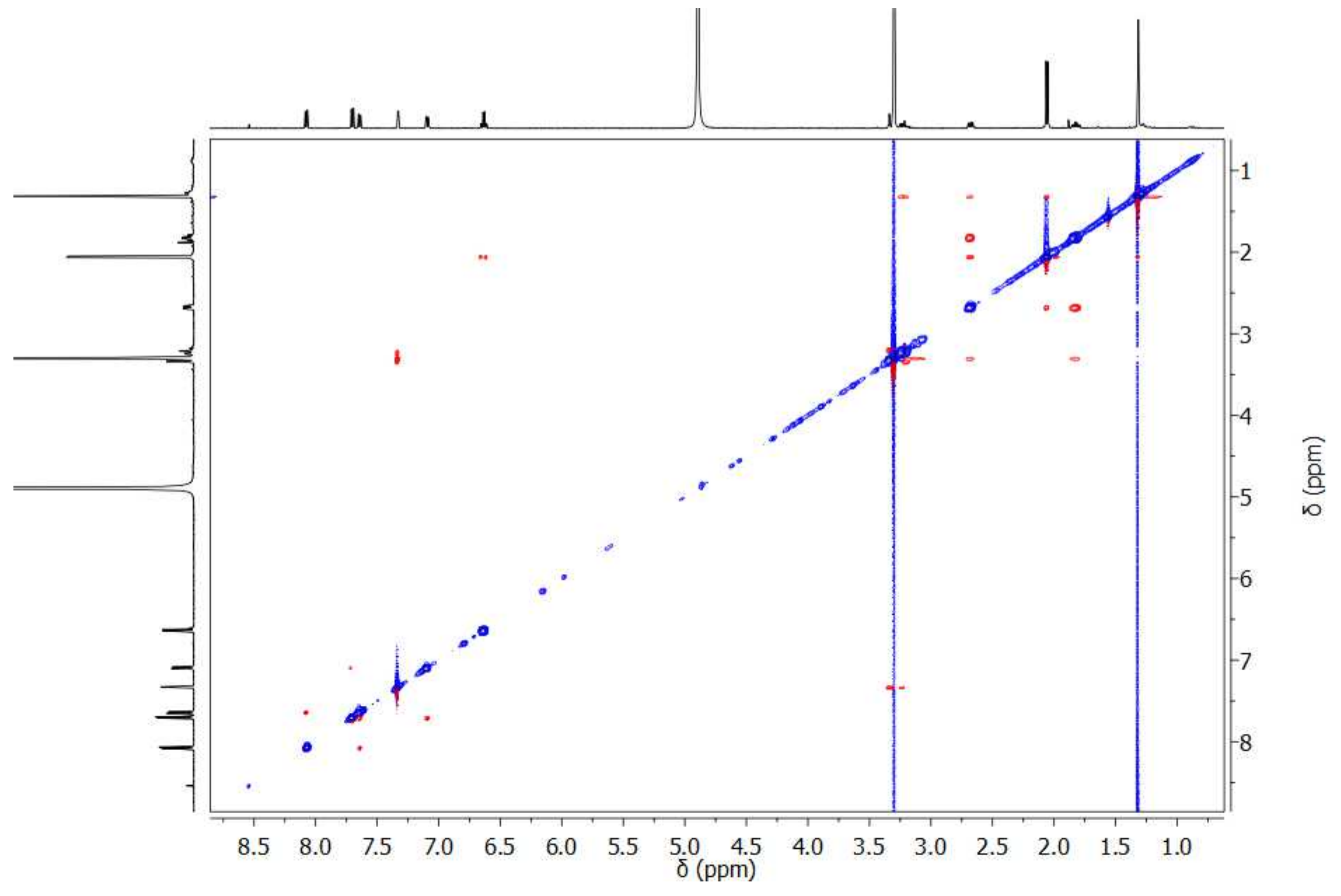


Figure S7. ${ }^{1} \mathrm{H}$ NMR Spectrum (500 MHz) of Cinanthrenol A (2) in MeOH- $d_{4}$

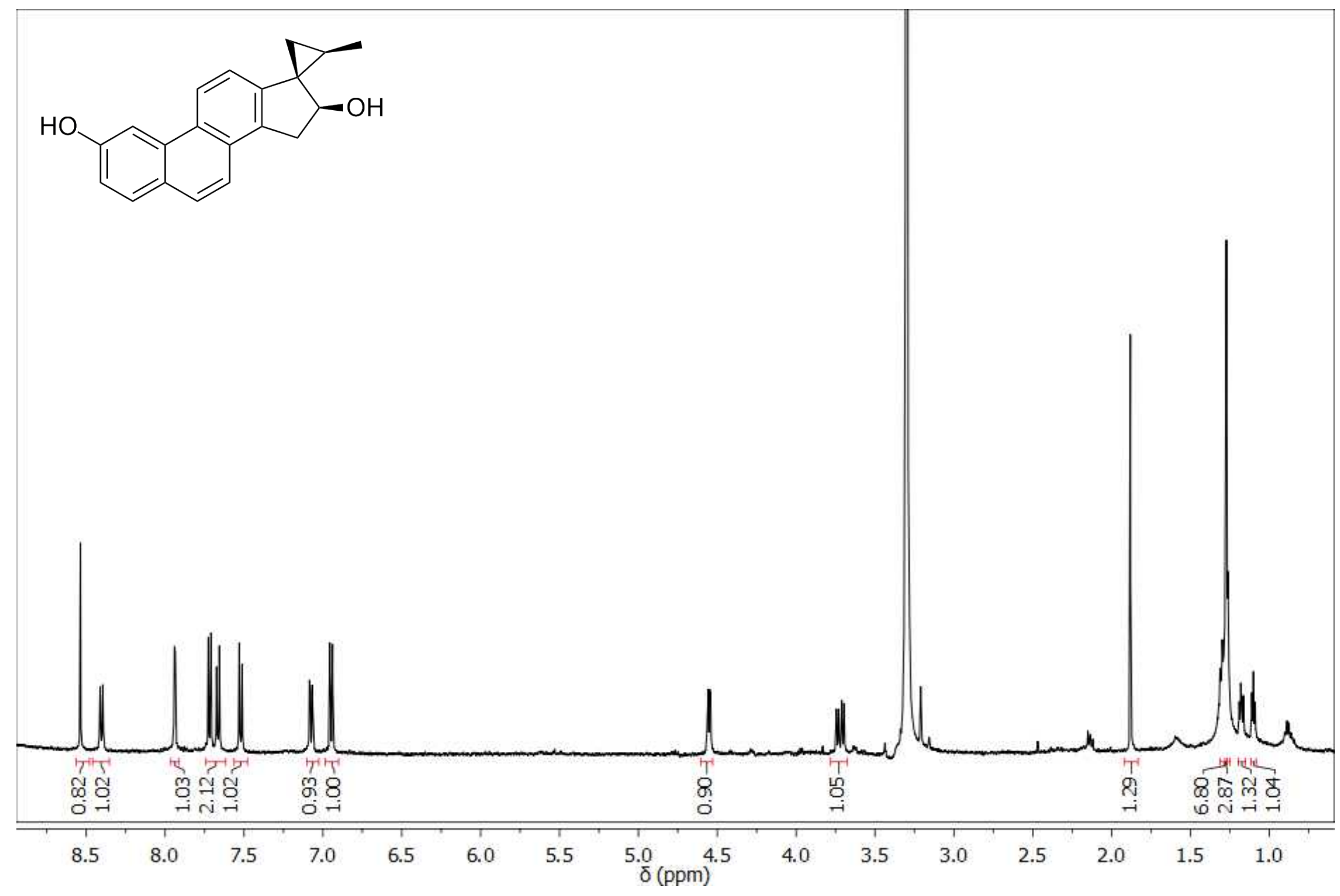


Figure S9. gHSQC NMR Spectrum (500 MHz) of Cinanthrenol A (2) in $\mathrm{MeOH}-d_{4}$

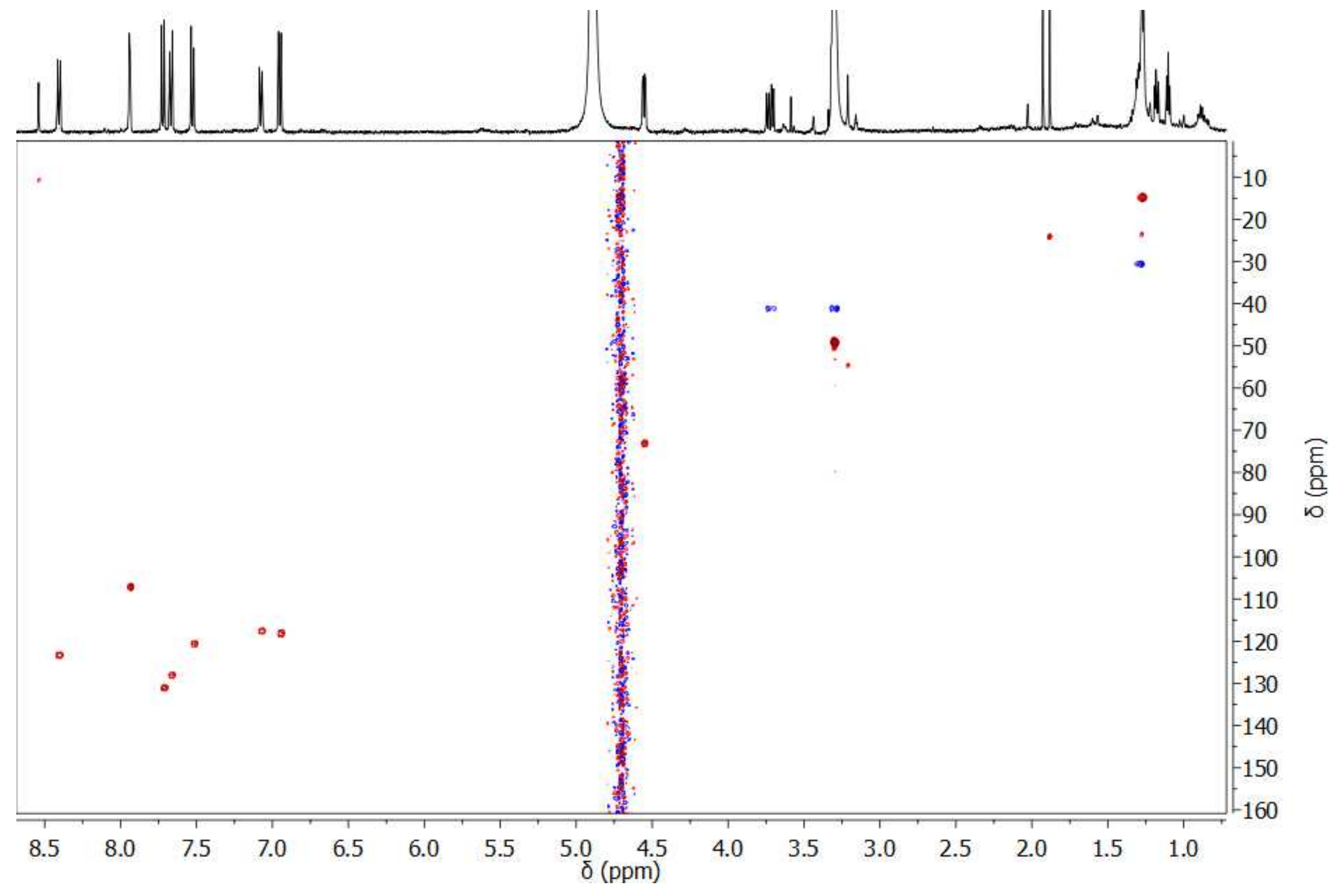




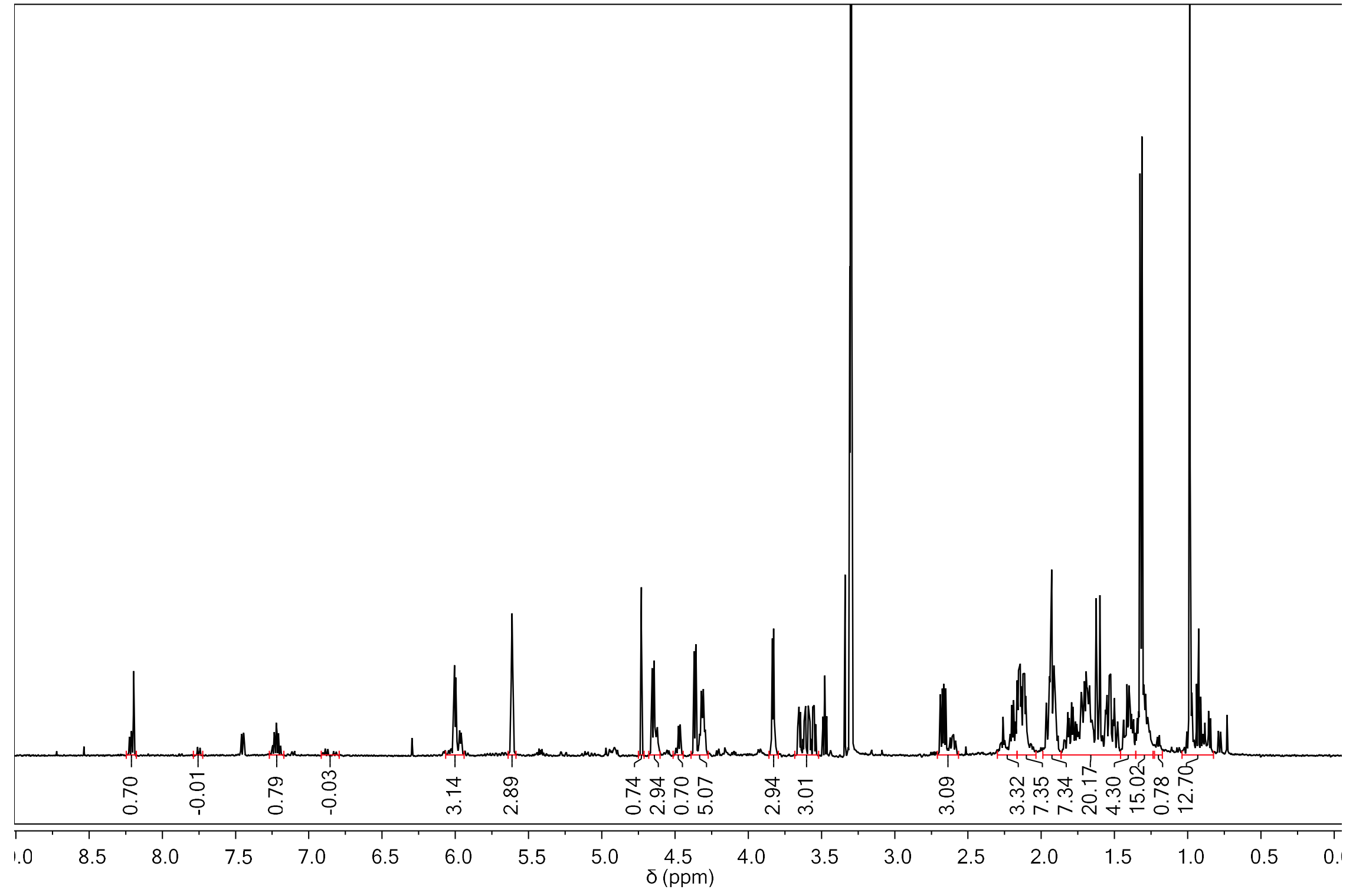


Figure 11: ${ }^{1} \mathrm{H}$ NMR Spectrum of 3,4-dihydroxypregna-5,20-diene-10,2-carbolactone (4) (CD3OD, $\left.500 \mathrm{MHz}\right)$

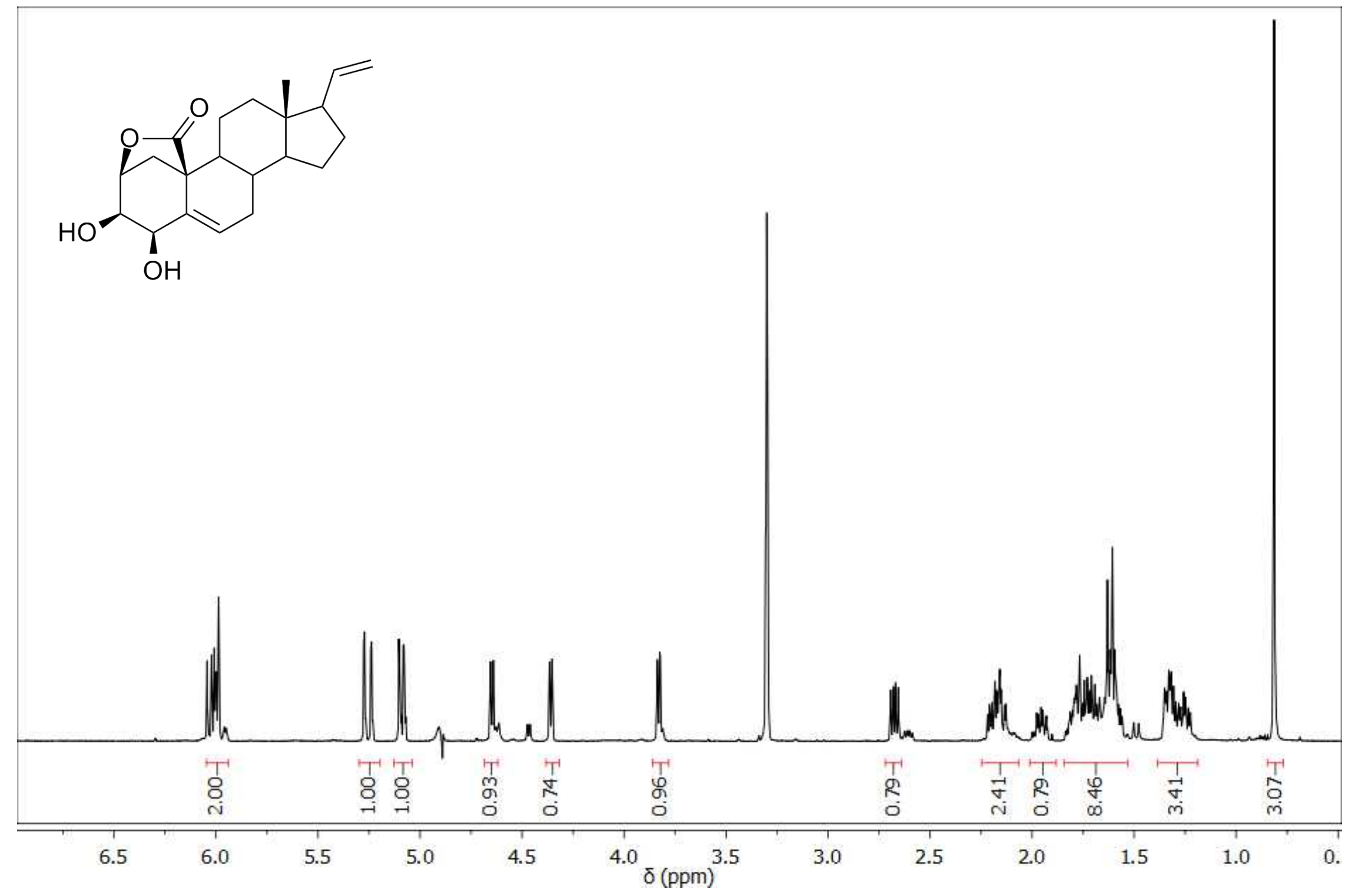


Figure S12. Image of Myrmekioderma sp.

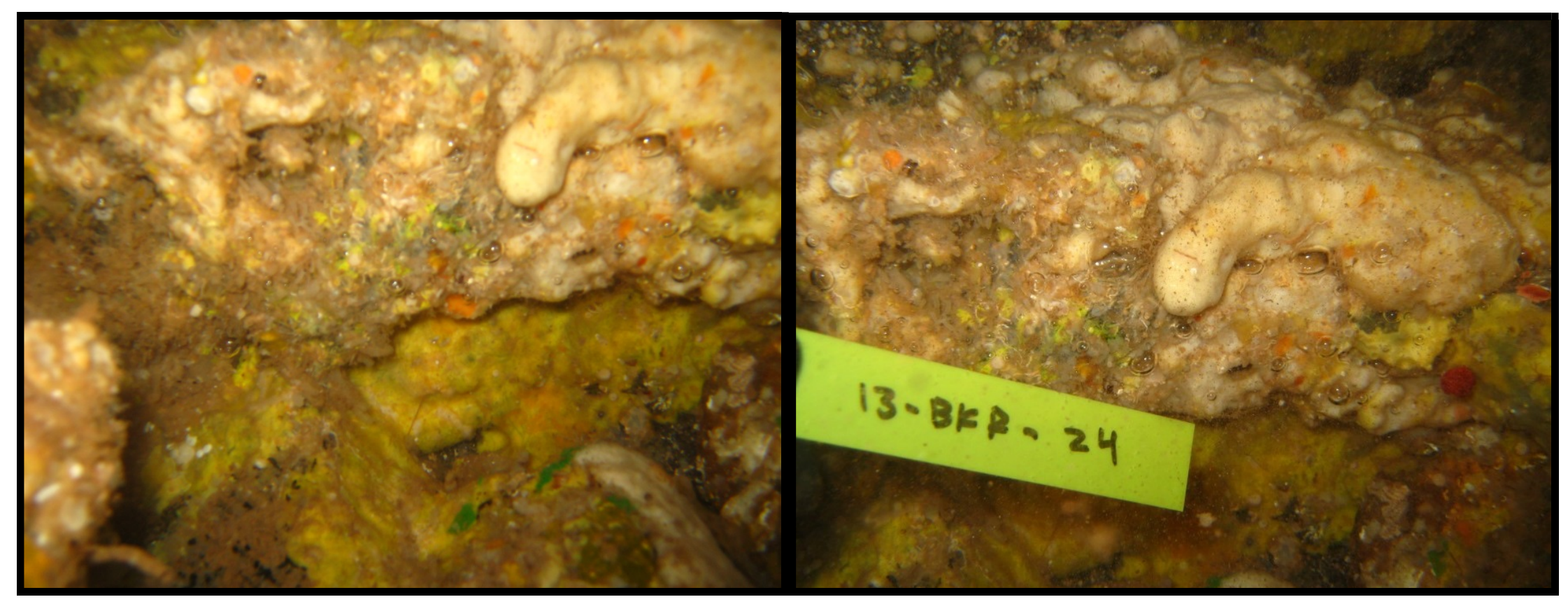


Table S1. Optimized Conformers Energy and Abundance.

\begin{tabular}{|c|c|c|c|c|c|}
\hline Filename & $\begin{array}{c}\text { Energy } \\
(\mathrm{kcal} / \mathrm{mol})\end{array}$ & $\begin{array}{c}\text { Relative Energy } \\
(\mathrm{kcal} / \mathrm{mol})\end{array}$ & Boltzmann Factor & $\begin{array}{c}\text { Equilibrium } \\
\text { Mole Fraction }\end{array}$ & $\begin{array}{c}\text { Number of Imaginary } \\
\text { Frequencies }\end{array}$ \\
\hline SP1-opt_freq-conf-1.out & -626268.4843 & 0.312499677 & 0.58976892 & 0.369809964 & 0 \\
\hline SP1-opt_freq-conf-3.out & -626265.1258 & 3.670929937 & 0.00202396 & 0.001269111 & 0 \\
\hline SP1-opt_freq-conf-4.out & -626265.358 & 3.438751463 & 0.00299626 & 0.001878782 & 0 \\
\hline SP1-opt_freq-conf-2.out & -626268.7968 & 0 & 1 & 0.627042143 & 0 \\
\hline
\end{tabular}

Conformer 1

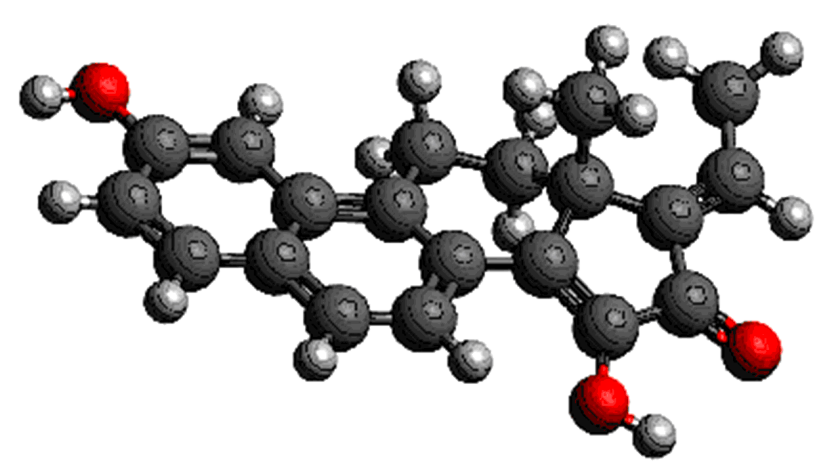

Conformer 3

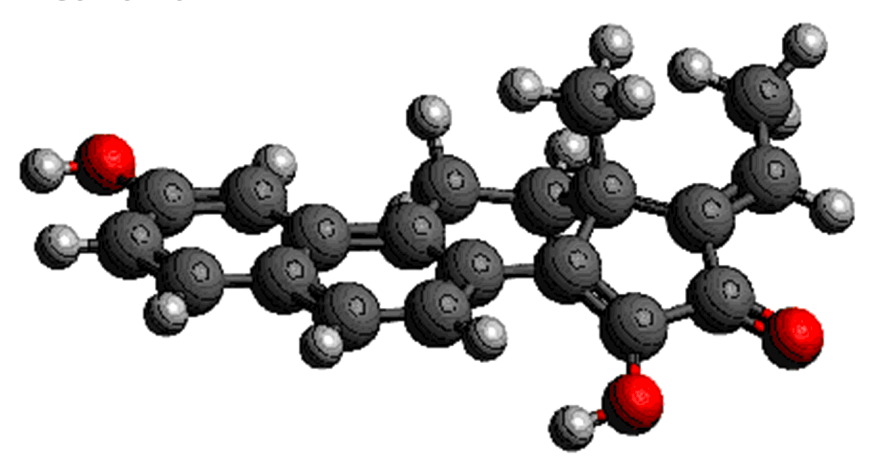

Conformer 2

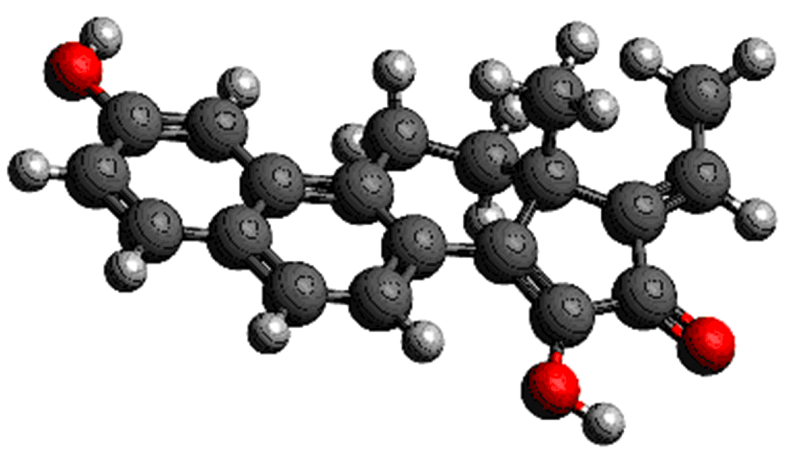

Conformer 4

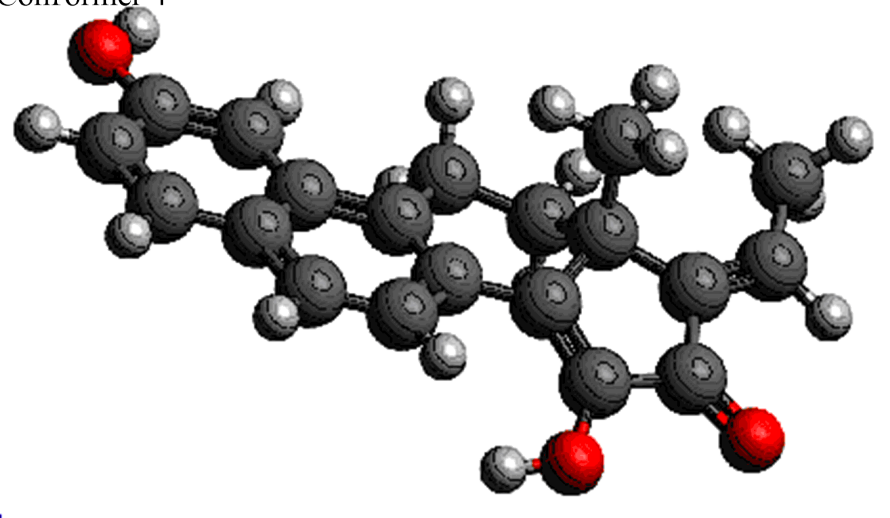


Table S2. Cartesian Coordinates For The Re-Optimized Conformer 1 Of Compound (1)

\begin{tabular}{|c|c|c|c|c|}
\hline Number & Atomic & \multicolumn{3}{|c|}{ Coordinates (Angstrom) } \\
\hline & & $\mathrm{X}$ & $\mathrm{Y}$ & $Z$ \\
\hline 1 & $\mathrm{C}$ & 4.330634 & 1.35584 & 0.206493 \\
\hline 2 & $\mathrm{C}$ & 2.920039 & 1.209219 & 0.161322 \\
\hline 3 & $\mathrm{C}$ & 2.364438 & -0.08278 & -0.07172 \\
\hline 4 & $\mathrm{C}$ & 3.245318 & -1.18449 & -0.23973 \\
\hline 5 & $\mathrm{C}$ & 4.609504 & -1.00656 & -0.18244 \\
\hline 6 & $\mathrm{C}$ & 5.164254 & 0.279162 & 0.038967 \\
\hline 7 & $\mathrm{C}$ & 2.056564 & 2.32343 & 0.347766 \\
\hline 8 & $\mathrm{C}$ & 0.699874 & 2.16344 & 0.314812 \\
\hline 9 & $\mathrm{C}$ & 0.129199 & 0.873948 & 0.088255 \\
\hline 10 & $\mathrm{C}$ & 0.939931 & -0.23685 & -0.12088 \\
\hline 11 & $\mathrm{C}$ & -1.32159 & 0.703284 & 0.076284 \\
\hline 12 & $\mathrm{C}$ & -1.90278 & -0.68947 & 0.284019 \\
\hline 13 & $\mathrm{C}$ & -1.14992 & -1.61185 & -0.68749 \\
\hline 14 & $\mathrm{C}$ & 0.355094 & -1.60631 & -0.39744 \\
\hline 15 & $\mathrm{C}$ & -3.37549 & -0.49427 & -0.03553 \\
\hline 16 & $\mathrm{C}$ & -3.60263 & 0.953716 & -0.26089 \\
\hline 17 & $\mathrm{C}$ & -2.29183 & 1.613533 & -0.16686 \\
\hline 18 & $\mathrm{O}$ & 5.403156 & -2.10009 & -0.34393 \\
\hline 19 & $\mathrm{O}$ & -2.17028 & 2.935868 & -0.39241 \\
\hline 20 & $\mathrm{O}$ & -4.64811 & 1.541486 & -0.51792 \\
\hline 21 & $\mathrm{C}$ & -4.38414 & -1.37112 & -0.11222 \\
\hline 22 & $\mathrm{C}$ & -4.33348 & -2.84978 & 0.100741 \\
\hline 23 & $\mathrm{C}$ & -1.71235 & -1.13002 & 1.748585 \\
\hline 24 & $\mathrm{H}$ & 4.748197 & 2.345111 & 0.38043 \\
\hline 25 & $\mathrm{H}$ & 2.867973 & -2.18775 & -0.40687 \\
\hline 26 & $\mathrm{H}$ & 6.245257 & 0.397598 & 0.076321 \\
\hline 27 & $\mathrm{H}$ & 2.495476 & 3.302709 & 0.525554 \\
\hline 28 & $\mathrm{H}$ & 0.038769 & 3.009417 & 0.469277 \\
\hline 29 & $\mathrm{H}$ & -1.3341 & -1.25861 & -1.70937 \\
\hline 30 & $\mathrm{H}$ & -1.523 & -2.63898 & -0.6276 \\
\hline 31 & $\mathrm{H}$ & 0.875785 & -2.05263 & -1.25175 \\
\hline 32 & $\mathrm{H}$ & 0.572636 & -2.26184 & 0.457755 \\
\hline 33 & $\mathrm{H}$ & 6.343921 & -1.85673 & -0.2859 \\
\hline 34 & $\mathrm{H}$ & -3.0586 & 3.295447 & -0.5893 \\
\hline 35 & $\mathrm{H}$ & -5.36149 & -0.95324 & -0.36204 \\
\hline 36 & $\mathrm{H}$ & -5.05793 & -3.13979 & 0.869319 \\
\hline
\end{tabular}




\begin{tabular}{|l|l|r|r|r|}
\hline 37 & H & -4.6253 & -3.37132 & -0.81774 \\
\hline 38 & H & -3.34833 & -3.20536 & 0.402129 \\
\hline 39 & H & -2.29165 & -0.48405 & 2.414367 \\
\hline 40 & H & -2.04824 & -2.1617 & 1.891158 \\
\hline 41 & H & -0.66145 & -1.06886 & 2.046008 \\
\hline
\end{tabular}

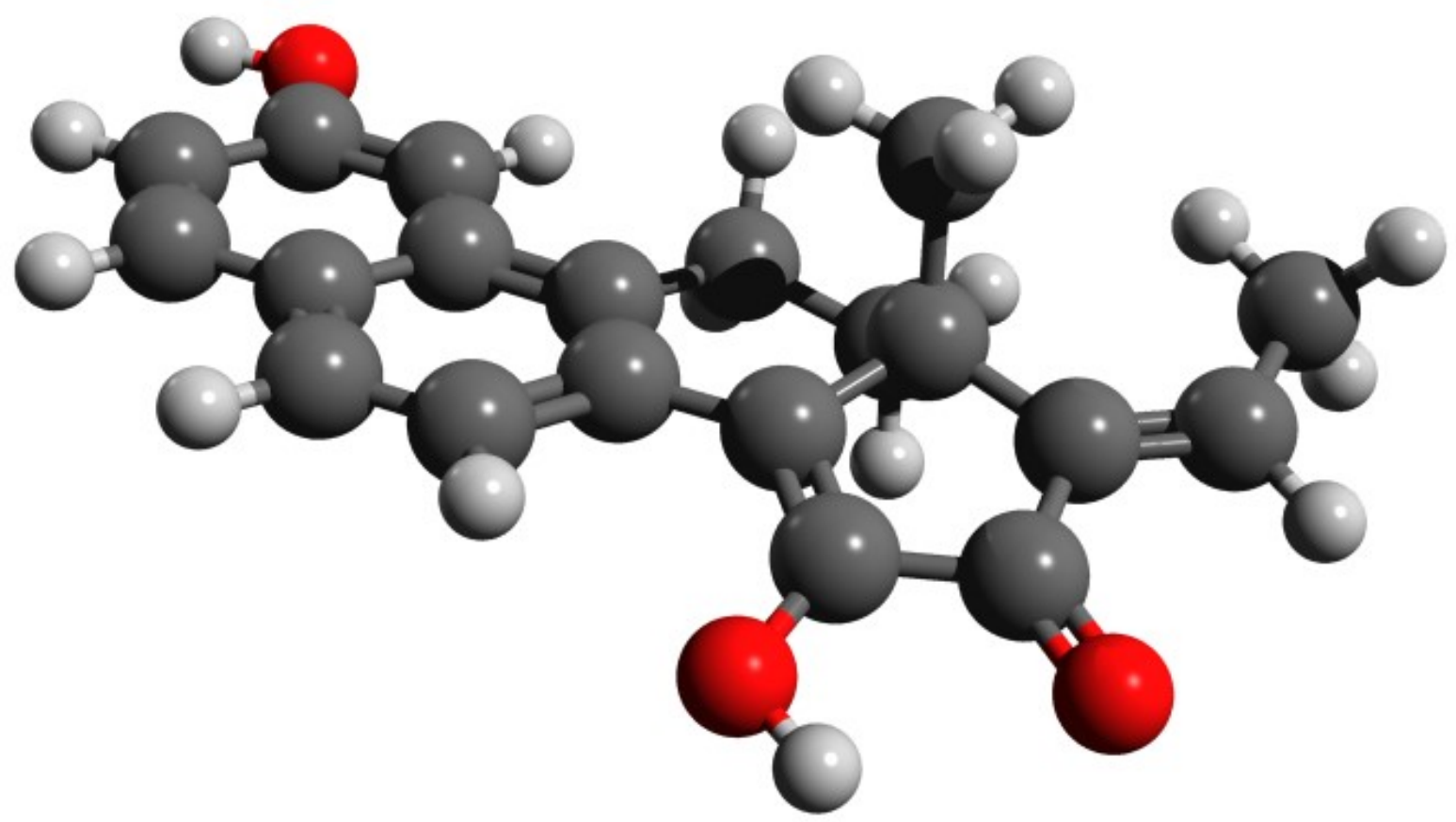


Table S3. Cartesian Coordinates For The Re-Optimized Conformer 2 Of Compound (1)

\begin{tabular}{|c|c|c|c|c|}
\hline Center & Atomic & \multicolumn{3}{|c|}{ Coordinates (Angstrom) } \\
\hline & & $\mathrm{X}$ & $\mathrm{Y}$ & $\mathrm{Z}$ \\
\hline 1 & $\mathrm{C}$ & 4.334731 & 1.360531 & 0.207675 \\
\hline 2 & $\mathrm{C}$ & 2.921933 & 1.212171 & 0.163804 \\
\hline 3 & $\mathrm{C}$ & 2.366905 & -0.0793 & -0.06781 \\
\hline 4 & $\mathrm{C}$ & 3.250959 & -1.18179 & -0.23717 \\
\hline 5 & $\mathrm{C}$ & 4.614208 & -0.99964 & -0.18302 \\
\hline 6 & $\mathrm{C}$ & 5.168335 & 0.286647 & 0.038684 \\
\hline 7 & $\mathrm{C}$ & 2.058616 & 2.32531 & 0.349629 \\
\hline 8 & $\mathrm{C}$ & 0.701209 & 2.165151 & 0.316369 \\
\hline 9 & $\mathrm{C}$ & 0.131627 & 0.876325 & 0.090813 \\
\hline 10 & $\mathrm{C}$ & 0.943854 & -0.2348 & -0.11656 \\
\hline 11 & $\mathrm{C}$ & -1.31901 & 0.704825 & 0.076975 \\
\hline 12 & $\mathrm{C}$ & -1.90008 & -0.68772 & 0.286223 \\
\hline 13 & $\mathrm{C}$ & -1.14574 & -1.61139 & -0.6828 \\
\hline 14 & $\mathrm{C}$ & 0.359097 & -1.60485 & -0.3915 \\
\hline 15 & $\mathrm{C}$ & -3.37239 & -0.49359 & -0.03611 \\
\hline 16 & $\mathrm{C}$ & -3.59945 & 0.953822 & -0.26518 \\
\hline 17 & $\mathrm{C}$ & -2.28907 & 1.614233 & -0.16993 \\
\hline 18 & $\mathrm{O}$ & 5.507 & -2.01395 & -0.33453 \\
\hline 19 & $\mathrm{O}$ & -2.16736 & 2.936048 & -0.39814 \\
\hline 20 & $\mathrm{O}$ & -4.64452 & 1.540701 & -0.52589 \\
\hline 21 & $\mathrm{C}$ & -4.38066 & -1.37087 & -0.11242 \\
\hline 22 & $\mathrm{C}$ & -4.32993 & -2.84899 & 0.10428 \\
\hline 23 & $\mathrm{C}$ & -1.71152 & -1.12562 & 1.751803 \\
\hline 24 & $\mathrm{H}$ & 4.74947 & 2.351039 & 0.381411 \\
\hline 25 & $\mathrm{H}$ & 2.862808 & -2.1828 & -0.40344 \\
\hline 26 & $\mathrm{H}$ & 6.24896 & 0.391884 & 0.071884 \\
\hline 27 & $\mathrm{H}$ & 2.497061 & 3.304915 & 0.526727 \\
\hline 28 & $\mathrm{H}$ & 0.040127 & 3.01131 & 0.470031 \\
\hline 29 & $\mathrm{H}$ & -1.32902 & -1.2601 & -1.70552 \\
\hline 30 & $\mathrm{H}$ & -1.51834 & -2.63862 & -0.62151 \\
\hline 31 & $\mathrm{H}$ & 0.879696 & -2.05252 & -1.24524 \\
\hline 32 & $\mathrm{H}$ & 0.575649 & -2.25919 & 0.464924 \\
\hline 33 & $\mathrm{H}$ & 5.04494 & -2.85917 & -0.47991 \\
\hline 34 & $\mathrm{H}$ & -3.05534 & 3.294941 & -0.59782 \\
\hline 35 & $\mathrm{H}$ & -5.35775 & -0.9539 & -0.36479 \\
\hline 36 & $\mathrm{H}$ & -5.05566 & -3.13733 & 0.87228 \\
\hline
\end{tabular}




\begin{tabular}{|l|l|r|r|r|}
\hline 37 & H & -4.6199 & -3.37288 & -0.81345 \\
\hline 38 & H & -3.34522 & -3.20347 & 0.408375 \\
\hline 39 & H & -2.29208 & -0.47871 & 2.415576 \\
\hline 40 & H & -2.04702 & -2.15722 & 1.895877 \\
\hline 41 & H & -0.66106 & -1.06323 & 2.050612 \\
\hline
\end{tabular}

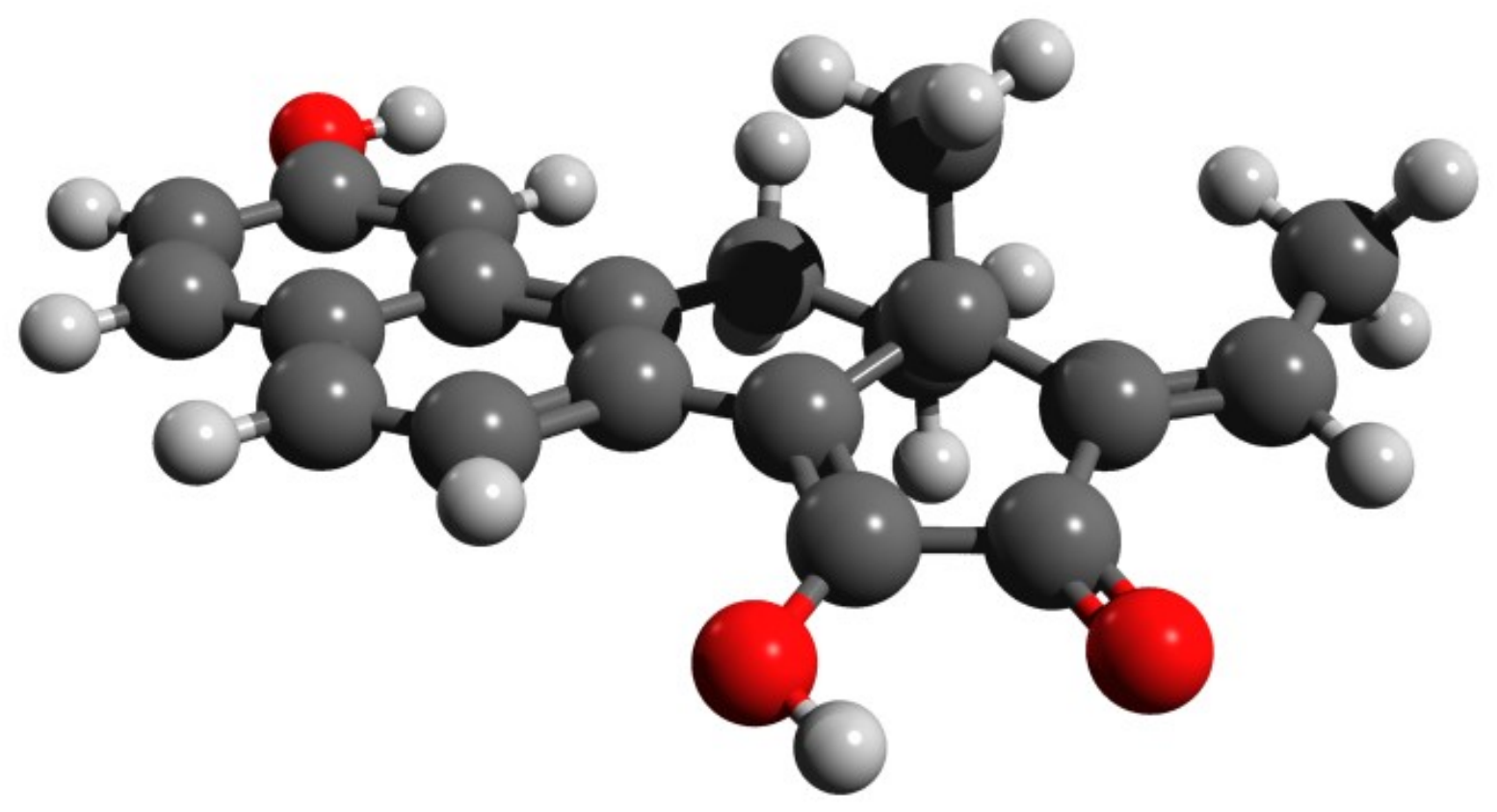


Table S4. Cartesian Coordinates For The Re-Optimized Conformer 3 Of Compound (1)

\begin{tabular}{|c|c|c|c|c|}
\hline Center & Atomic & \multicolumn{3}{|c|}{ Coordinates (Angstrom) } \\
\hline & & $\mathrm{X}$ & $\mathrm{Y}$ & $\mathrm{Z}$ \\
\hline 1 & $\mathrm{C}$ & 4.328564 & 1.315938 & 0.317626 \\
\hline 2 & $\mathrm{C}$ & 2.917104 & 1.18307 & 0.251108 \\
\hline 3 & $\mathrm{C}$ & 2.353511 & -0.08502 & -0.07677 \\
\hline 4 & $\mathrm{C}$ & 3.227601 & -1.17854 & -0.31546 \\
\hline 5 & $\mathrm{C}$ & 4.592686 & -1.01444 & -0.23794 \\
\hline 6 & $\mathrm{C}$ & 5.155254 & 0.248055 & 0.078658 \\
\hline 7 & $\mathrm{C}$ & 2.061265 & 2.283121 & 0.525994 \\
\hline 8 & $\mathrm{C}$ & 0.703582 & 2.135086 & 0.464518 \\
\hline 9 & $\mathrm{C}$ & 0.124293 & 0.878847 & 0.113535 \\
\hline 10 & $\mathrm{C}$ & 0.928324 & -0.22446 & -0.14782 \\
\hline 11 & $\mathrm{C}$ & -1.32992 & 0.718657 & 0.058769 \\
\hline 12 & $\mathrm{C}$ & -1.9092 & -0.66952 & 0.291554 \\
\hline 13 & $\mathrm{C}$ & -1.18068 & -1.58894 & -0.70458 \\
\hline 14 & $\mathrm{C}$ & 0.335299 & -1.58039 & -0.47149 \\
\hline 15 & $\mathrm{C}$ & -3.38417 & -0.47224 & -0.0043 \\
\hline 16 & $\mathrm{C}$ & -3.61094 & 0.966777 & -0.30803 \\
\hline 17 & $\mathrm{C}$ & -2.28473 & 1.616973 & -0.27347 \\
\hline 18 & $\mathrm{O}$ & 5.37952 & -2.09795 & -0.4742 \\
\hline 19 & $\mathrm{O}$ & -2.21911 & 2.920436 & -0.61848 \\
\hline 20 & $\mathrm{O}$ & -4.67042 & 1.518156 & -0.57057 \\
\hline 21 & $\mathrm{C}$ & -4.39861 & -1.34497 & -0.02143 \\
\hline 22 & $\mathrm{C}$ & -4.35172 & -2.81487 & 0.247429 \\
\hline 23 & $\mathrm{C}$ & -1.68236 & -1.11 & 1.749195 \\
\hline 24 & $\mathrm{H}$ & 4.751354 & 2.286809 & 0.566265 \\
\hline 25 & $\mathrm{H}$ & 2.844913 & -2.16405 & -0.55837 \\
\hline 26 & $\mathrm{H}$ & 6.236761 & 0.355091 & 0.131779 \\
\hline 27 & $\mathrm{H}$ & 2.503145 & 3.237507 & 0.803206 \\
\hline 28 & $\mathrm{H}$ & 0.056962 & 2.967624 & 0.731655 \\
\hline 29 & $\mathrm{H}$ & -1.4048 & -1.23723 & -1.71876 \\
\hline 30 & $\mathrm{H}$ & -1.54796 & -2.6176 & -0.63124 \\
\hline 31 & $\mathrm{H}$ & 0.827728 & -1.9893 & -1.36073 \\
\hline 32 & $\mathrm{H}$ & 0.588291 & -2.26704 & 0.348863 \\
\hline 33 & $\mathrm{H}$ & 6.321852 & -1.86712 & -0.39279 \\
\hline 34 & $\mathrm{H}$ & -1.31592 & 3.181819 & -0.86857 \\
\hline 35 & $\mathrm{H}$ & -5.37934 & -0.93024 & -0.26301 \\
\hline 36 & $\mathrm{H}$ & -5.00721 & -3.06079 & 1.090264 \\
\hline
\end{tabular}




\begin{tabular}{|l|l|r|r|r|}
\hline 37 & H & -4.73452 & -3.36642 & -0.61819 \\
\hline 38 & H & -3.34905 & -3.17784 & 0.472745 \\
\hline 39 & H & -2.23171 & -0.45423 & 2.430727 \\
\hline 40 & H & -2.0293 & -2.13573 & 1.904732 \\
\hline 41 & H & -0.62206 & -1.06528 & 2.014803 \\
\hline
\end{tabular}

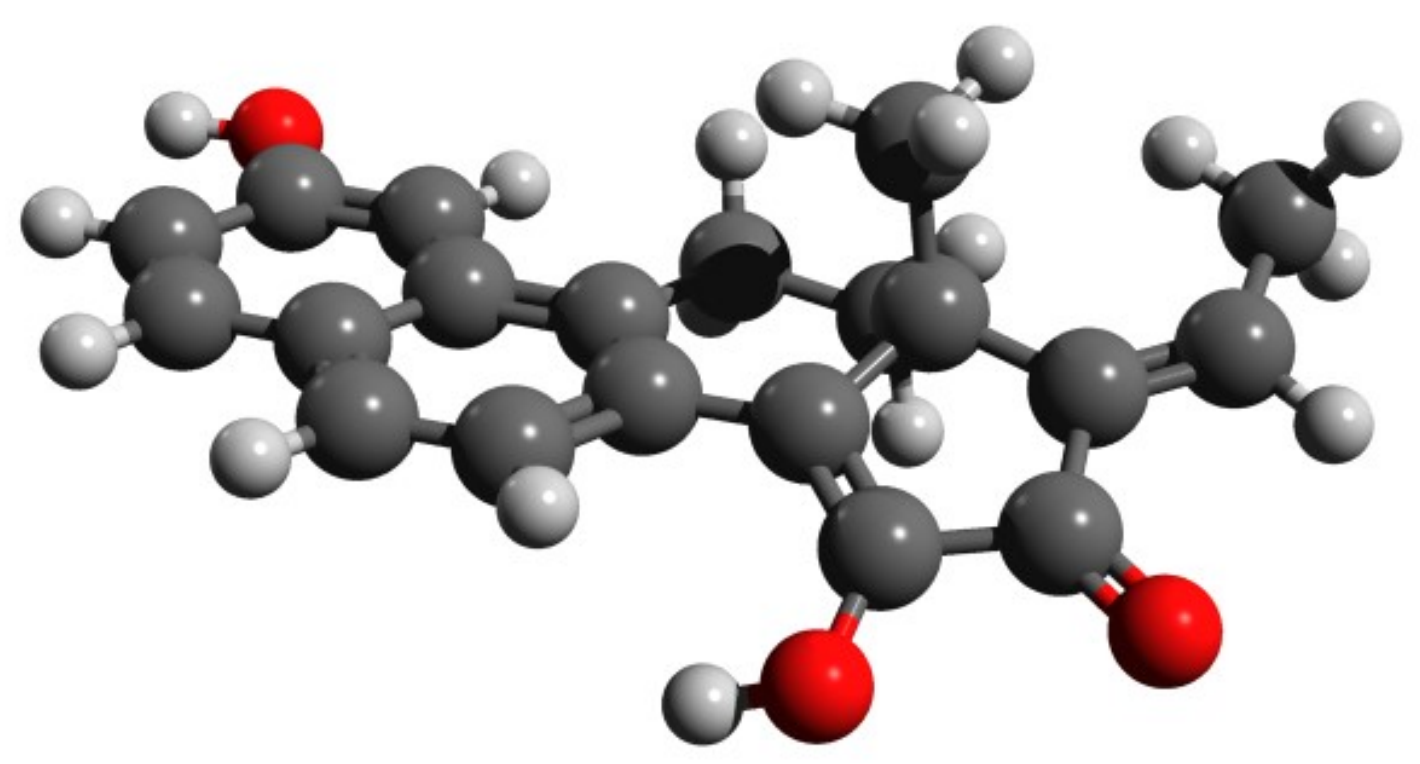


Table S5. Cartesian Coordinates For The Re-Optimized Conformer 4 Of Compound (1)

\begin{tabular}{|c|c|c|c|c|}
\hline Center & Atomic & \multicolumn{3}{|c|}{ Coordinates (Angstrom) } \\
\hline & & $\mathrm{X}$ & $\mathrm{Y}$ & $\mathrm{Z}$ \\
\hline 1 & $\mathrm{C}$ & 4.332664 & 1.320747 & 0.317601 \\
\hline 2 & $\mathrm{C}$ & 2.919007 & 1.185667 & 0.252734 \\
\hline 3 & $\mathrm{C}$ & 2.356049 & -0.0823 & -0.07266 \\
\hline 4 & $\mathrm{C}$ & 3.233355 & -1.17687 & -0.31145 \\
\hline 5 & $\mathrm{C}$ & 4.597547 & -1.00801 & -0.23702 \\
\hline 6 & $\mathrm{C}$ & 5.159381 & 0.255611 & 0.078333 \\
\hline 7 & $\mathrm{C}$ & 2.063425 & 2.284892 & 0.526314 \\
\hline 8 & $\mathrm{C}$ & 0.705013 & 2.136606 & 0.465089 \\
\hline 9 & $\mathrm{C}$ & 0.126817 & 0.880715 & 0.116032 \\
\hline 10 & $\mathrm{C}$ & 0.932291 & -0.2232 & -0.14328 \\
\hline 11 & $\mathrm{C}$ & -1.32723 & 0.719961 & 0.059355 \\
\hline 12 & $\mathrm{C}$ & -1.90669 & -0.66776 & 0.293762 \\
\hline 13 & $\mathrm{C}$ & -1.1767 & -1.58877 & -0.69981 \\
\hline 14 & $\mathrm{C}$ & 0.339105 & -1.57974 & -0.46515 \\
\hline 15 & $\mathrm{C}$ & -3.38131 & -0.47114 & -0.00487 \\
\hline 16 & $\mathrm{C}$ & -3.60748 & 0.967093 & -0.31261 \\
\hline 17 & $\mathrm{C}$ & -2.28144 & 1.61756 & -0.27656 \\
\hline 18 & $\mathrm{O}$ & 5.484528 & -2.01393 & -0.4553 \\
\hline 19 & $\mathrm{O}$ & -2.21502 & 2.920333 & -0.62384 \\
\hline 20 & $\mathrm{O}$ & -4.66639 & 1.517718 & -0.57901 \\
\hline 21 & $\mathrm{C}$ & -4.39597 & -1.34362 & -0.02127 \\
\hline 22 & $\mathrm{C}$ & -4.35001 & -2.81288 & 0.251492 \\
\hline 23 & $\mathrm{C}$ & -1.68163 & -1.10523 & 1.752535 \\
\hline 24 & $\mathrm{H}$ & 4.752532 & 2.293196 & 0.565044 \\
\hline 25 & $\mathrm{H}$ & 2.839828 & -2.16038 & -0.55225 \\
\hline 26 & $\mathrm{H}$ & 6.240452 & 0.349766 & 0.126044 \\
\hline 27 & $\mathrm{H}$ & 2.504953 & 3.239918 & 0.80183 \\
\hline 28 & $\mathrm{H}$ & 0.058476 & 2.969596 & 0.730992 \\
\hline 29 & $\mathrm{H}$ & -1.39959 & -1.23885 & -1.71487 \\
\hline 30 & $\mathrm{H}$ & -1.54384 & -2.61738 & -0.62521 \\
\hline 31 & $\mathrm{H}$ & 0.831548 & -1.99034 & -1.35368 \\
\hline 32 & $\mathrm{H}$ & 0.590724 & -2.26516 & 0.356716 \\
\hline 33 & $\mathrm{H}$ & 5.018922 & -2.84411 & -0.66337 \\
\hline 34 & $\mathrm{H}$ & -1.31149 & 3.181 & -0.87349 \\
\hline 35 & $\mathrm{H}$ & -5.37619 & -0.92928 & -0.26554 \\
\hline 36 & $\mathrm{H}$ & -5.00588 & -3.05612 & 1.094815 \\
\hline
\end{tabular}




\begin{tabular}{|l|l|l|l|l|}
\hline 37 & H & -4.73284 & -3.36652 & -0.61276 \\
\hline 38 & H & -3.34765 & -3.17582 & 0.478146 \\
\hline 39 & H & -2.23266 & -0.44871 & 2.431972 \\
\hline 40 & H & -2.02764 & -2.13103 & 1.909665 \\
\hline 41 & H & -0.62177 & -1.05876 & 2.019698 \\
\hline
\end{tabular}

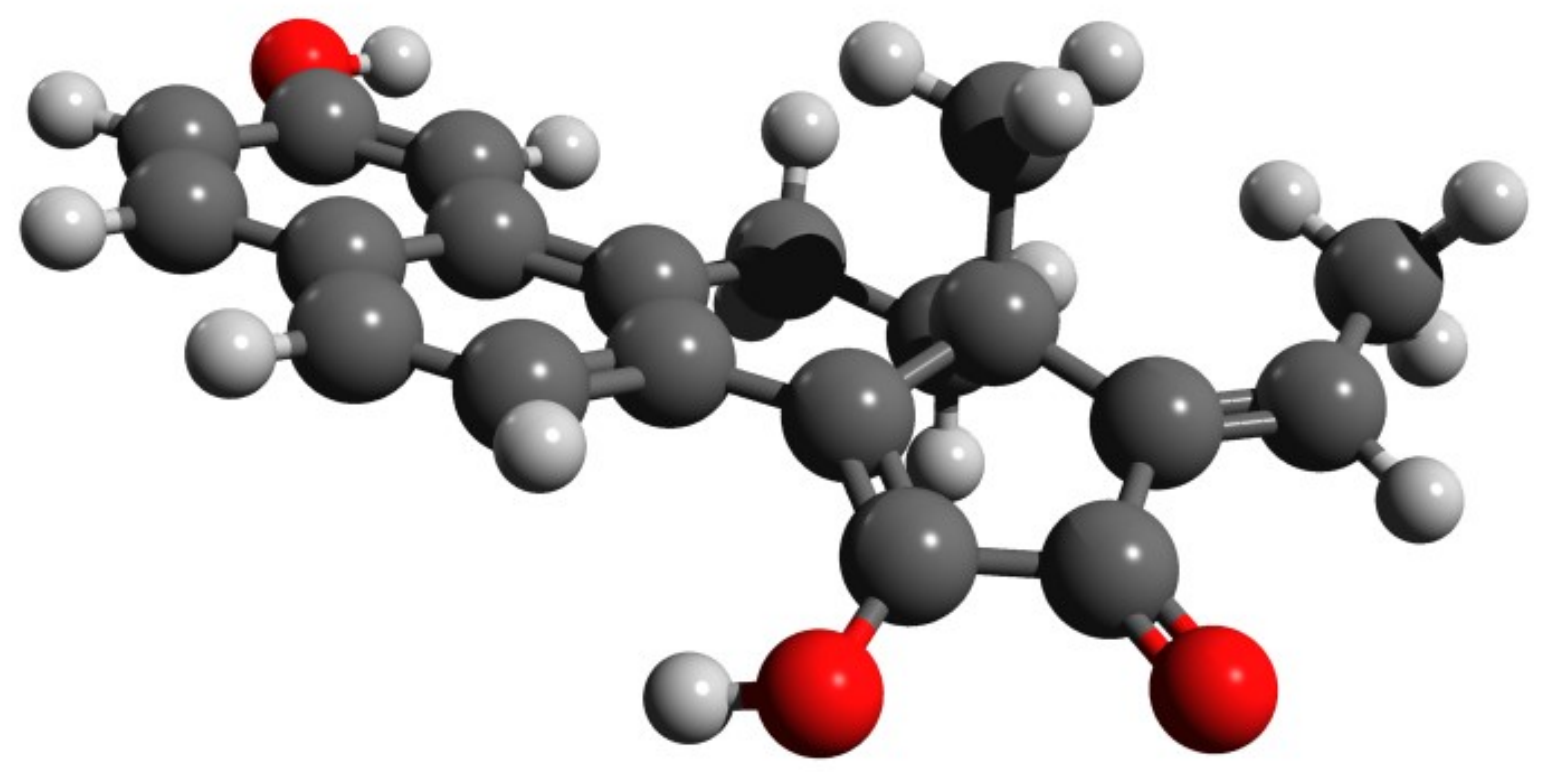

